CDD: 001.535

\title{
EXPRESSIVENESS AND DEFINABILITY IN CIRCUMSCRIPTION
}

\author{
FRANCICLEBER MARTINS FERREIRA ${ }^{1}$ \\ ANA TERESA MARTINS ${ }^{2}$ \\ Departamento de Computação \\ Universidade Federal do Ceará \\ Fortaleza, CE, CP 12.166 \\ $B R A S I L$
}

fran@lia.ufc.br,ana@lia.ufc.br

\begin{abstract}
We investigate expressiveness and definability issues with respect to minimal models, particularly in the scope of Circumscription. First, we give a proof of the failure of the Löwenheim-Skolem Theorem for Circumscription. Then we show that, if the class of $\bar{P} ; \bar{Z}$-minimal models of a first-order sentence is $\Delta$ elementary, then it is elementary. That is, whenever the circumscription of a firstorder sentence is equivalent to a first-order theory, then it is equivalent to a finitely axiomatizable one. This means that classes of models of circumscribed theories are either elementary or not $\Delta$-elementary. Finally, using the previous result, we prove that, whenever a relation $P_{i}$ is defined in the class of $\bar{P} ; \bar{Z}$-minimal models of a firstorder sentence $\phi$ and whenever such class of $\bar{P} ; \bar{Z}$-minimal models is $\Delta$-elementary, then there is an explicit definition $\psi$ for $P_{i}$ such that the class of $\bar{P} ; \bar{Z}$-minimal models of $\phi$ is the class of models of $\phi \wedge \psi$. In order words, the circumscription of $\bar{P}$ in $\phi$ with $\bar{Z}$ varied can be replaced by $\phi$ plus this explicit definition $\psi$ for $P_{i}$.
\end{abstract}

Keywords: Minimal models. Circumscripition. Expressiveness. Definability.

\section{INTRODUCTION}

In practical situations, people reason and act without having complete or sufficient knowledge about the situation that they are dealing

\footnotetext{
${ }^{1}$ This author was partially supported by CNPq, FUNCAP, CAPES (DS, PROCAD 2010).

${ }^{2}$ This author was partially supported by CNPq (PQ, Casadinho 2008, Universal 2010) and CAPES (PROCAD 2010).
}

Manuscrito - Rev. Int. Fil., Campinas, v. 34, n. 1, p. 233-266, jan.-jun. 2011. 
with. Sometimes, there is no way or it is too much expensive to obtain all the necessary information in order to be secure about our conclusions. However, in such cases, we may find ourselves in a position in which it is mandatory to take some action or make some inferences. For instance, a pilot which need to abort landing the airplane for some technical problem just discovered will immediately go-around instead of waiting for the confirmation that this action will not lead to a crash with another aircraft. Reasoning under such circumstances is, thus, required. Any system intended to describe or simulate practical reasoning must be able to deal with the lack of information. Actually, even in daily activities we assume beliefs which we take as certain, but for which there is no logical, deductive justification (Hume (1748)). However, although we cannot deduce from earlier facts that to put ours hands on the fire will burn them, no mentally healthy person will do this and think that nothing will go wrong.

The lack of information and the need of drawing conclusions force us to go beyond of which can be deduced from our partial, current knowledge. In real life, we make use of general or uncertain knowledge, such as that "birds generally flies" and that penguins do not, to guide us in the task of making such assumptions. However, due to the character of uncertainty of such sort of assumption, it may be confronted with new, reliable information and be refuted. Hence, besides the ability of handling the lack of information and the use of general and uncertain knowledge, another feature of a system that models practical reasoning is to be able to backtrack and drop some previous conclusions inferred from uncertain assumptions in order to stay consistent with the new information obtained.

In order to formalize practical reasoning under partial knowledge, classical logic is not appropriate. In fact, classical logic is deductive and, as a deductive approach to reasoning, classical logic cannot go beyond and infer more than what is already known. As we argued 
above, in practical situations one must conclude more than what can be deduced, that is, a logical system for practical reasoning must allow non-deductive inferences. Another feature of classical reasoning is the monotonicity property: the addition of new premises does not invalidate previous inferences. On the contrary, logical systems for practical reasoning must be non-monotonic, since conclusions taken under partial knowledge may be defeated. Hence, classical logic is not suited to properly deal with practical reasoning.

In the 1980s, with the increasing interest in Artificial Intelligence, some non-monotonic logical systems where proposed to formalize practical reasoning, such as Reiter's Default logic Reiter (1980), Doyle and McDermott's Non-Monotonic logic McDermott, Doyle (1980) and McCarthy's Circumscription McCarthy $(1980,1986)$. Our focus, here, is in the latter approach since we are interested in minimal models, as we explain below.

McCarthy's Predicate Circumscription is one of the most studied logical approaches to non-monotonic reasoning (see Lifschitz (1994) for a good introduction and extensive bibliography). In McCarthy (1980), McCarthy introduces Circumscription to deal with the Qualification Problem. The Qualification Problem is the problem of describing or qualifying the necessary conditions to take an action or safely infer some information in a given situation. As argued in McCarthy (1980), it is practically impossible to deal with the huge amount of constraints or conditions necessary for the success of an action. In practice, people disregard many possible obstacles or assumptions contrary to some conclusion just because they are unknown, there is no evidence for it, or they usually do not happen. Usually, we concentrate on the relevant evidences drawing conclusions even in the absence of information about conditions of success. We also suppose, under the lack of knowledge about the consequences of some action, that things not directly involved in the action will stand as they were before the action was 
taken. McCarthy argued also that if, on the one hand, we must go beyond what can be deduced from the known information, on the other hand we should avoid unreasonable assumptions. That is the case, for instance, of the winged horse solution for McCarthy's Missionaries and Cannibals puzzle McCarthy (1980). General or default knowledge is used as an heuristics, a guide to reasoning.

The intuitive idea of Predicate Circumscription is to consider that the objects which have some property are only those necessary to satisfy the problem description. In McCarthy (1980), Predicate Circumscription was introduced as a first-order formula schema and, in McCarthy (1986), as a second-order formula, with an additional extension called Formula Circumscription. Here, we will call them first-order Circumscription and second-order Circumscription, respectively. Predicate Circumscription works minimizing the extent of some relation in the problem description. A variant called Parallel Circumscription works minimizing a tuple of relations at the same time. In a model of a circumscribed theory, the circumscribed relations are intended to have extents as minimal as possible and satisfying the theory. Such models are called minimal models.

Minimal models were used in the 1980s to provide semantics for McCarthy's Circumscription McCarthy $(1980,1986)$. In the 1980s and mid 1990s, the mathematics of minimal models was widely studied in the scope of Circumscription and its extensions (see Lifschitz (1994)). In 1995, Lifschitz extended McCarthy's Circumscription to Nested Abnormality Theories (NATs) Lifschitz (1995). In NATs, circumscriptions can be grouped into blocks and circumscribed again. Recent work has been done on the computational complexity of satisfiability and validity of propositional versions of NATs Cadoli et al (2005). In Ferreira, Martins (2007), the logical expressiveness of NATs, as presented in Lifschitz (1995), was studied and it was proved that the expressive power 
of NATs is equivalent to the expressive power of second-order logic, modulo extra relations.

Minimal models are also related with fixed-points. In van Benthem (2005), van Benthem introduced the MIN(FO) logic that allows one to define the minimal relation which satisfies certain syntactically defined sort of formulas: the PIA-Conditions. These minimal relations are the extent of $P$ in the $P$-minimal models (see Section 2 for the definition of $P$-minimal model) of the corresponding PIA-Condition, for a syntactically determined relation symbol $P$ in the PIA-Condition. van Benthem (2005) showed that MIN(FO) is equivalent to the Least Fixed Point Logic - LFP (see Dawar, Gurevich (2002) for results on LFP).

In this paper, we will examine expressiveness and definability properties of logics with respect to minimal models, in particular, Circumscription. In Section 2, we will introduce the basic concepts of Minimal Model Theory and Circumscription. In Section 3, we will investigate the expressive power of Circumscription. First, we will give a proof that the Löwenheim-Skolem Theorem does not hold for Circumscription. After that, we will prove a theorem relating classes of minimal models and $\Delta$-elementary classes (see Definition 3.6). We will show that, if the class of $\bar{P} ; \bar{Z}$-minimal models of a first-order sentence is $\Delta$-elementary, then it is elementary. That is, whenever a circumscribed theory is equivalent to a first-order theory, then it is equivalent to a finitely axiomatizable one. It follows that classes of minimal models of first-order sentences either are elementary or are not even $\Delta$-elementary. In Section 4, we investigate definability on Circumscription. We will introduce the main concepts of the Theory of Definitions and address a statement made in Doyle (1985) regarding definitions and Circumscription. We will also state and prove a theorem which can be taken as an alternative approach to some problems faced in Doyle (1985). We will show that, whenever the circumscription of a tuple of relations $\bar{P}$ in a finite first-order theory $\phi$, implicitly defines 
some circumscribed relation $P_{i}$ and such circumscription is equivalent to a (possibly countable) first-order theory $\Gamma$, then there is a specific explicit definition $\psi$ for $P_{i}$ in $\Gamma$ which, together with the initial theory $\phi$, is equivalent to the theory $\Gamma$. We will use the result of Section 3 to show that the theory $\Gamma$ is finitely axiomatizable. In Section 5 , we will draw some conclusions about all we have done in the previous sections.

The basic logic notation used throughout this text follows that in Ebbinghaus et al (1994). For instance, a symbol set $S$ is a set of relation, function and constant symbols. An $S$-structure is a pair $\mathfrak{A}=(A, \sigma)$, where $A$ is a set and $\sigma$ is a map which associates an $n$-ary relation $\sigma(P)=P^{\mathfrak{A}} \subset A^{n}$ to each $n$-ary relation symbol $P \in S$, an $n$-ary function $\sigma(f)=f^{\mathfrak{A}}: A^{n} \rightarrow A$ to each $n$-ary function symbol $f \in S$ and an element $\sigma(c)=c^{\mathfrak{A}} \in A$ to each constant symbol $c \in S$. (We use "relation" instead of "relation symbol" when the meaning is clear from the context.) The satisfiability relation $\models$ is defined as usually, for instance as in Ebbinghaus et al (1994), for both first-order and second-order sentences. We use Fraktur capital letters, such as $\mathfrak{A}, \mathfrak{B}, \mathfrak{C}, \ldots$, to denote structures and the corresponding Roman capital letters $A, B, C, \ldots$ for their domains. We call $\operatorname{Mod}_{S}(\Gamma)$ the class of $S$-structures which satisfy (are models of) the $S$-sentences in the set $\Gamma$ of $S$-sentences. Given a class of $S$-structures $\mathbb{C}$, we call $\operatorname{Th}_{S}^{F O}(\mathbb{C})$ the set of first-order $S$-sentences satisfied by every $S$-structure in $\mathbb{C}$.

In the next section, we will introduce the main concepts of Minimal Model Theory and Circumscription that will be used in Sections 3 and 4 . 


\section{MINIMAL MODELS AND CIRCUMSCRIPTION}

By minimal models we mean those defined over the following relation.

Definition 2.1 $[\mathfrak{A} \leq \bar{P} ; \bar{Z} \mathfrak{B}]$ Let $S \cup\{\bar{P}, \bar{Z}\}$ be a symbol set such that $\bar{P}=P_{1}, \ldots, P_{m}$ and $\bar{Z}=Z_{1}, \ldots, Z_{v}$ are tuples of relation symbols. Let $\mathfrak{A}$ and $\mathfrak{B}$ be two structures on the symbol set $S \cup\{\bar{P}, \bar{Z}\}$ (in other words, two $S \cup\{\bar{P}, \bar{Z}\}$-structures) with the same domain $A$. We say that $\mathfrak{A}$ and $\mathfrak{B}$ agree on the interpretation of all symbols in $S$ iff, for each $s \in S$, we have

$$
s^{\mathfrak{A}}=s^{\mathfrak{B}} .
$$

We define the relation $\leq^{\bar{P} ; \bar{Z}}$ between structures on the same symbol set $S \cup\{\bar{P}, \bar{Z}\}$ which agree on $S$ and with varied $\bar{Z}$ as:

$$
\mathfrak{A} \leq \bar{P} ; \bar{Z} \mathfrak{B} \text { iff, for each } 1 \leq i \leq m, P_{i}^{\mathfrak{A}} \subseteq P_{i}^{\mathfrak{B}} .
$$

When the tuple $\bar{Z}$ has length $0(\bar{Z}=\emptyset)$, we write $\leq^{\bar{P}}$ for $\leq^{\bar{P} ; \emptyset}$.

It is easy to see that $\leq \bar{P} ; \bar{Z}$ is a preorder, that is, it is reflexive and transitive. Hereafter, we consider $\bar{P}=P_{1}, \ldots, P_{m}$ and $\bar{x}_{i}$ a tuple of variable whose length is equal to the arity of $P_{i}, 1 \leq i \leq m, \bar{Z}=$ $Z_{1}, \ldots, Z_{v}$ and $y_{j}$ is a tuple of variables whose length is equal to the arity of $Z_{j}, 1 \leq j \leq v$. We also consider $S,\{\bar{P}\}$ and $\{\bar{Z}\}$ as pairwise disjoint and $S^{\prime}=S \cup\{\bar{P}\} \cup\{\bar{Z}\}$, if not otherwise stated. Also note that we use a semi-colon between the tuple of relations $\bar{P}$ and the tuple of varied relations $\bar{Z}$ instead of a comma, which is used between symbols in a tuple or list. If $\bar{Z}$ is empty, then the relation $\leq \bar{P}$ is a partial order, that is, an antisymmetric preorder. An example of this case occurs when $S^{\prime}=\{\bar{P}\}$, which means that all relation symbols in the symbol set are minimized.

Definition 2.2 $[\bar{P} ; \bar{Z}$-Minimal Models of $\mathbb{C}]$ Let $\mathbb{C}$ be a class of $S \cup\{\bar{P}, \bar{Z}\}$-structures. A structure $\mathfrak{A} \in \mathbb{C}$ is a $\bar{P} ; \bar{Z}$-minimal model of 
$\mathbb{C}$ iff $\mathfrak{A}$ is minimal with respect to the restriction of $\leq \bar{P} ; \bar{Z}$ to $\mathbb{C}$, that is, there is no $\mathfrak{B} \in \mathbb{C}$ such that

$$
\mathfrak{B} \leq \leq^{\bar{P} ; \bar{Z}} \mathfrak{A} \text { and not } \mathfrak{A} \leq \leq^{\bar{P} ; \bar{Z}} \mathfrak{B}
$$

When $\bar{Z}$ has length 0 , we say that $\mathfrak{A} \in \mathbb{C}$ is a $\bar{P}$-minimal model instead of $\bar{P} ; \emptyset$-minimal model. Given an $S^{\prime}$-sentence $\phi$, we say that a model is a $\bar{P} ; \bar{Z}$-minimal model of $\phi$ if it is a $\bar{P} ; \bar{Z}$-minimal model of $\operatorname{Mod}_{S^{\prime}}(\phi)$.

Definition 2.3 [Minimal Consequence $\models \bar{P} ; \bar{Z}$ ] Given two sentences $\phi$ and $\psi$, we say that $\phi \bar{P} ; \bar{Z}$-minimally entails $\psi$, in symbols $\phi \models \bar{P} ; \bar{Z} \psi$, iff each $\bar{P} ; \bar{Z}$-minimal model of $\phi$ is a model of $\psi$.

McCarthy's Circumscription captures the Closed World Assumption Reiter (1978) by means of minimal models. In McCarthy (1980), McCarthy introduced Circumscription as a first-order formula schema. Let $T(\bar{P}, \bar{Z})$ be a first-order sentence on the symbol set $S \cup\{\bar{P}, \bar{Z}\}$, where $\bar{P}=P_{1}, \ldots, P_{m}$ and $\bar{Z}:=Z_{1}, \ldots, Z_{v}$ are tuples of relation symbols. The circumscription schema for $\bar{P}$ in $T(\bar{P}, \bar{Z})$ with varied $\bar{Z}$ is the following expression:

$$
\begin{gathered}
C S(T(\bar{P}, \bar{Z}) ; \bar{\Phi} ; \bar{\zeta}) \\
:= \\
\left(T(\bar{\Phi}, \bar{\zeta}) \wedge \bigwedge_{1 \leq i \leq m} \forall \bar{x}_{i}\left(\Phi_{i}\left(\bar{x}_{i}\right) \rightarrow P_{i}\left(\bar{x}_{i}\right)\right)\right) \rightarrow \bigwedge_{1 \leq i \leq m} \forall \bar{x}_{i}\left(P_{i}\left(\bar{x}_{i}\right) \rightarrow \Phi_{i}\left(\bar{x}_{i}\right)\right),
\end{gathered}
$$

where $\bar{\Phi}=\Phi_{1}, \ldots, \Phi_{m}$ is a tuple of metavariables standing for any $S \cup\{\bar{P}, \bar{Z}\}$-formulas $\bar{\phi}=\phi_{1}\left(\bar{x}_{1}\right), \ldots, \phi_{m}\left(\bar{x}_{m}\right)$ with free variables in $\bar{x}_{i}$, respectively, and such that the length of $\bar{x}_{i}$ is equal to the arity of $P_{i}$, and $\bar{\zeta}$ is a tuple of metavariables standing for any tuple of $S \cup\{\bar{P}, \bar{Z}\}$ formulas $\bar{\delta}:=\delta_{1}\left(\bar{y}_{1}\right), \ldots, \delta_{v}\left(\bar{y}_{v}\right)$ such that the length of $\bar{y}_{i}$ is equal to the arity of $Z_{i}$. An instance of (1) is obtained by replacing every occurrence of $P_{i}(\bar{t})$ with $\phi_{i}(\bar{t})$, for any tuple $\bar{t}=t_{1}, \ldots, t_{n}$ of terms in the language, 
and every occurrence of $Z_{i}(\bar{t})$ with $\delta_{i}(\bar{t})$, for any tuple $\bar{t}$ of terms in the language, with the appropriate substitutions of bound variables in the formulas $\phi_{1}\left(\bar{x}_{1}\right), \ldots, \phi_{m}\left(\bar{x}_{m}\right), \delta_{1}\left(\bar{y}_{1}\right), \ldots, \delta_{v}\left(\bar{y}_{v}\right)$ to avoid undesired biding of the variables in $\bar{t}$. Such an instance says that, if the relations defined by $\phi_{1}\left(\bar{x}_{1}\right), \ldots, \phi_{m}\left(\bar{x}_{m}\right), \delta_{1}\left(\bar{y}_{1}\right), \ldots, \delta_{v}\left(\bar{y}_{v}\right)$ satisfy the sentence $T(\bar{\phi}, \bar{\delta})$ and the relation $P_{i}$ contains the relation defined by $\phi_{i}\left(\bar{x}_{i}\right)$, for each $1 \leq i \leq m$, then the relation defined by $\phi_{i}\left(\bar{x}_{i}\right)$ also contains the relation $P_{i}$ for $1 \leq i \leq m$. Hence, the schema (1) says that, every definable relation that satisfies $T$ and is contained in $P_{i}$, also contains $P_{i}$, with $\bar{Z}$ varying among definable relations. We define first-order circumscription as follows.

Definition 2.3 [First-Order Circumscription] Given a sentence $T(\bar{P}, \bar{Z})$, we define the first-order circumscription of $\bar{P}$ in $T(\bar{P}, \bar{Z})$ with varied $\bar{Z}$ as the set of sentences

$$
\operatorname{Circ}_{F O}[T(\bar{P}, \bar{Z}) ; \bar{P} ; \bar{Z}]:=\{T(\bar{P}, \bar{Z})\} \cup\{\theta \mid \theta \text { is an instance of }(1)\} \text {. }
$$

We write $\operatorname{Circ}_{F O}[T(\bar{P}, \bar{Z}) ; \bar{P}]$ for $\operatorname{Circ}_{F O}[T(\bar{P}, \bar{Z}) ; \bar{P} ; \emptyset]$.

Any $\bar{P} ; \bar{Z}$-minimal model of $T(\bar{P}, \bar{Z})$ is a model of $\operatorname{Circ}_{F O}[T(\bar{P}$, $\bar{Z}) ; \bar{P} ; \bar{Z}]$. However, it can be proved that, for some $S \cup\{\bar{P}, \bar{Z}\}$-sentence $T(\bar{P} ; \bar{Z})$, not every model of $\operatorname{Circ}_{F O}[T(\bar{P} ; \bar{Z}) ; \bar{P} ; \bar{Z}]$ is a $\bar{P} ; \bar{Z}$-minimal model in the sense of Definition 2.2. For instance, consider the sentence

$T(P):=\forall x \forall y(E(x, y) \rightarrow P(x, y)) \wedge \forall x \forall y \forall z(P(x, y) \wedge E(y, z) \rightarrow P(x, z))$.

The first conjunct says that the relation $P$ contains the relation $E$ and second conjunct says that $P$ is closed with respect to $E$. In other words, $P$ contains the transitive closure of $E$. It is easy to see that in any $P$-minimal model $\mathfrak{A}$ of $T(P), P^{\mathfrak{A}}$ is exactly the transitive closure of $E^{\mathfrak{A}}$. However, there is no first-order theory which axiomatizes the 
class of structures $\mathfrak{A}=\left(A, E^{\mathfrak{A}}, P^{\mathfrak{A}}\right)$ where $P^{\mathfrak{A}}$ is the transitive closure of $E^{\mathfrak{A}}$.

In McCarthy (1986), McCarthy reformulated his Circumscription and captured precisely the minimal models semantics with respect to the relation $\leq \bar{P} ; \bar{Z}$ of Definition 2.1. McCarthy presented Circumscription as a second-order formula.

Definition 2.5 [Second-Order Circumscription] Given a firstorder sentence $T(\bar{P}, \bar{Z})$, the second-order circumscription of the relations $\bar{P}=P_{1}, \ldots, P_{m}$ in $T(\bar{P}, \bar{Z})$ with varied $\bar{Z}$, represented by Circ $_{S O}$ $[T(\bar{P}, \bar{Z}) ; \bar{P} ; \bar{Z}]$, is defined as the following second-order formula:

$$
\begin{gathered}
\operatorname{Circ}_{S O}[T(\bar{P}, \bar{Z}) ; \bar{P} ; \bar{Z}] \\
:= \\
T(\bar{P}, \bar{Z}) \wedge \forall \bar{X} \forall \overline{Z^{\prime}}\left(T\left(\bar{X}, \overline{Z^{\prime}}\right) \rightarrow \neg(\bar{X} \subsetneq \bar{P})\right),
\end{gathered}
$$

where

$$
\bar{X} \subsetneq \bar{P}:=\bar{X} \subseteq \bar{P} \wedge \neg(\bar{X}=\bar{P})
$$

and $\bar{X} \subseteq \bar{P}:=\bigwedge_{1 \leq i \leq m} \forall \bar{x}_{i}\left(X_{i}\left(\bar{x}_{i}\right) \rightarrow P_{i}\left(\bar{x}_{i}\right)\right)$ and $\bar{X}=\bar{P}:=\bar{X} \subseteq$ $\bar{P} \wedge \bar{P} \subseteq \bar{X}$. If there is no varied relation $(\bar{Z}=\emptyset)$, we write $\operatorname{Circ}_{S O}[T$ $(\bar{P}, \bar{Z}) ; \bar{P}]$ instead of $\operatorname{Circ}_{S O}[T(\bar{P}, \bar{Z}) ; \bar{P} ; \emptyset]$.

A proof of the following statement can be found in Lifschitz (1994):

Theorem 2.6 The $\bar{P} ; \bar{Z}$-minimal models of $T(\bar{P} ; \bar{Z})$ are exactly the models of

$$
\operatorname{Circ}_{S O}[T(\bar{P} ; \bar{Z}) ; \bar{P} ; \bar{Z}]
$$

It is easy to see from Theorem 2.6 above that $\operatorname{Circ}_{S O}[T(\bar{P} ; \bar{Z}) ; \bar{P}$; $\bar{Z}] \models \phi$ iff $T(\bar{P}, \bar{Z}) \models_{\bar{P} ; \bar{Z}} \phi$. In the next section, we will give a characterization of the classes of $\bar{P} ; \bar{Z}$-minimal models of first-order sentences. This result will be used in the Section 4 . 


\section{TWO EXPRESSIVENESS THEOREMS}

The expressive power of a logic is its capability to express classes of structures or to distinguish between classes of structures. Our aim in this section is to give a characterization of the expressive power of second-order Circumscription. This is achieved by Theorems 3.5 and 3.7 and Corollary 3.8 below. As we will see, the definability results in the next section will appear as applications of the results obtained in this section.

In Schlipf(1987), Schlipf studied decidability questions regarding Circumscription such as to decide whether a first-order formula has a countable minimal model or not. Among other things, Schlipf showed that some formulas which have minimal models do not have countable minimal models Example 2.6 in Schlipf(1987). It means that an analogue to the Downward Löwenheim-Skolem Theorem does not hold for second-order Circumscription.

Theorem 3.1 [Schlipf] There is a first-order formula $\phi$ which has only uncountable minimal models.

This fact is particularly important in our characterization of the expressive power of first- and second-order Circumscription. In order to keep this paper self-contained, we will give a proof, different from that of Schlipf, of the failure of the Downward Löwenheim-Skolem Theorem for Circumscription. Schlipf's proof is based on the existence of $\omega_{1}$-like models of Peano Arithmetic. These $\omega_{1}$-like models are models of cardinality $\aleph_{1}$, but each element in these models has only countable many predecessor (see Schlipf(1987), p.177). Then, Schlipf uses second-order Circumscription in order to avoid countable models.

Our proof is based on another set-theoretical fact, namely that continuous dense linear orderings are uncountable. A dense, linear ordering $<$ of a set $A$ is continuous iff any subset $A^{\prime} \subset A$ which has a upper bound in $A$ with respect to $<$, also has a least upper bound with respect 
to $<$. We use just two relation symbols. Let $<$ be a binary relation symbol and $P$ a unary relation symbol. Let $L O(<)$ be a first-order sentence saying that $<$ is a strict, dense, linear order without the least element:

$$
\begin{aligned}
L O(<):= & \forall x(\neg x<x) \wedge \forall x \forall z(x<z \rightarrow \exists y(x<y \wedge y<z)) \wedge \\
& \forall x \forall y \forall z(x<y \wedge y<z \rightarrow x<z) \wedge \\
& \forall x \forall y(x<y \vee x=y \vee y<x) \wedge \\
& \neg \exists x \forall y(x<y \vee x=y),
\end{aligned}
$$

and consider the following formulas:

$$
\begin{aligned}
U B(P, x) & :=\forall y(P(y) \rightarrow(y<x \vee y=x)), \\
L U B(P) & :=\exists x(U B(P, x) \wedge \forall y(U B(P, y) \rightarrow(x<y \vee x=y))), \\
D(P) & :=\exists x(U B(P, x)) \rightarrow L U B(P), \\
D C(P) & :=\forall x(P(x) \rightarrow \forall y(y<x \rightarrow P(y))), \\
N E(P) & :=\exists x(P(x)) .
\end{aligned}
$$

$D(P)$ says that, if there is an upper bound for the elements in $P$, then there is a least one. We call $D(P)$ the Dedekind property for $P$. $D C(P)$ says that $P$ is downward closed, that is, if an element $x$ belongs to $P$, then any other element less than $x$ with respect to $<$ also belongs to $P$. The intuitive meaning of the other formulas is obvious.

Let

$$
T(P):=L O(<) \wedge D C(P) \wedge N E(P) \wedge \neg D(P) .
$$


It is easy to see, using Definition 2.5, that

$$
\begin{gathered}
\operatorname{Circ}_{S O}[T(P) ; P] \\
\equiv \\
T(P) \wedge \forall P^{\prime}\left(\left(D C\left(P^{\prime}\right) \wedge N E\left(P^{\prime}\right) \wedge P^{\prime} \subsetneq P\right) \rightarrow D\left(P^{\prime}\right)\right) .
\end{gathered}
$$

Intuitively, $\operatorname{Circ}_{S O}[T(P) ; P]$ says that any downward closed, proper subset $P^{\prime}$ of $P$ has the Dedekind property, that is, any downward closed proper subset of $P$ which has an upper bound also has a least upper bound. However, by $T(P), P$ itself does not have the Dedekind property.

Lemma 3.2 $\operatorname{Circ}_{S O}[T(P) ; P]$ is satisfiable.

Proof. Let $\mathfrak{R}=\left(R,<^{\mathfrak{R}}\right)$ be the structure of the real numbers with order and $\mathfrak{Q}=\left(Q,<^{\mathfrak{Q}}\right)$ be an isomorphic copy of the structure of the rational numbers with order disjoint from $\mathfrak{R}$ (that is, $R \cap Q=\emptyset$ ). Let $\mathfrak{B}^{\prime}=\left(B^{\prime},<^{\mathfrak{B}^{\prime}}\right)$ be the disjoint union of $\mathfrak{R}$ and $\mathfrak{Q}$, that is

$$
B^{\prime}=R \cup Q \text { and }<^{\mathfrak{B}^{\prime}}=\left(<^{\mathfrak{R}} \cup<^{\mathfrak{Q}}\right) .
$$

Now, let $\mathfrak{B}=\left(B,<^{\mathfrak{B}}\right)$ be obtained from $\mathfrak{B}^{\prime}$ by defining

$$
B=B^{\prime} \text { and }<^{\mathfrak{B}}=\left(<^{\mathfrak{B}^{\prime}} \cup(R \times Q)\right) .
$$

That is, any element in $R$ is less than each element in $Q$ with respect to $<^{\mathfrak{B}}$. If we imagine a graphical representation of the ordering $<^{\mathfrak{B}}$ where the increasing order is from left to right, the real numbers appears as a continuous ordering on the left and the rationals as a dense ordering on the right. Now, let 


$$
\mathfrak{A}=\left(\mathfrak{B}, P^{\mathfrak{A}}\right)
$$

with $P^{\mathfrak{A}}=R$. It is not too much difficult to check that $\mathfrak{A}$ is a model for $\operatorname{Circ}_{S O}[T(P) ; P]$.

We will need the following well known result of Set Theory and the Theory of Order Hrbacek, Jech (1999) in order to prove Theorem 3.5. Recall that a linear order without end-points is an order without the least or the greatest elements.

Theorem 3.3 Any two countable, dense, linearly ordered sets without end-points are order-isomorphic.

The following lemma will lead to the failure of the LöwenheimSkolem Theorem for Circumscription.

Lemma 3.4 Any model of $\operatorname{Circ}_{S O}[T(P) ; P]$ is uncountable.

Proof. In any model $\mathfrak{A}=\left(A,<^{\mathfrak{A}}, P^{\mathfrak{A}}\right)$ of $\operatorname{Circ}_{S O}[T(P) ; P],<^{\mathfrak{A}}$ is a dense linear ordering of the domain $A$, and $P^{\mathfrak{A}}$ is a nonempty, downward closed subset of $A$. As $A$ does not have a least element with respect to $<^{\mathfrak{A}}$ (see $\left.L O(<)\right), P^{\mathfrak{A}}$ has infinitely many elements. Since $\mathfrak{A} \models \neg D(P)$, it follows that $P^{\mathfrak{A}}$ does not have a greatest element. Let $P^{\prime \prime \mathfrak{A}}$ be any proper subset of $P^{\mathfrak{A}}$ with an upper bound in $P^{\mathfrak{A}}$ and

$$
P^{\prime \mathfrak{A}}=\left\{a \in A \mid \text { exists } b \in P^{\prime / \mathfrak{A}} \text { such that } a<^{\mathfrak{A}} b \text { or } a=b\right\} .
$$

$P^{\prime \mathfrak{A}}$ is a downward closed, proper subset of $P^{\mathfrak{A}}$. Moreover, any upper bound for $P^{\prime \prime \mathfrak{A}}$ is an upper bound for $P^{\prime \mathfrak{A}}$ and vice-versa. Thus $P^{\prime \mathfrak{A}}$ and $P^{\prime \prime \mathfrak{A}}$ have the same least upper bound, if any. As $\mathfrak{A} \models \operatorname{Circ}_{S O}[T(P) ; P]$ and $P^{\prime \mathfrak{A}} \subsetneq P^{\mathfrak{A}}$, it follows that $P^{\prime \mathfrak{A}}$ has a least upper bound in $A$. But, by definition, $P^{\prime / \mathfrak{A}}$, and therefore $P^{\prime \mathfrak{A}}$, has an upper bound in $P^{\mathfrak{A}}$. As $P^{\mathfrak{A}}$ is downward closed, the least upper bound of $P^{\prime \mathfrak{A}}$ belongs to $P^{\mathfrak{A}}$. Therefore, $P^{\prime \prime \mathfrak{A}}$ has a least upper bound in $P^{\mathfrak{A}}$. 
We get that any proper subset of $P^{\mathfrak{A}}$ with an upper bound in $P^{\mathfrak{A}}$ has a least upper bound in $P^{\mathfrak{A}}$. It follows that the substructure $\mathfrak{C}=\left(C,<^{\mathfrak{C}}\right)$ obtained by defining $C=P^{\mathfrak{A}}$ and $<^{\mathfrak{C}}$ as the restriction of $<^{\mathfrak{A}}$ to $P^{\mathfrak{A}}$, that is,

$$
<^{\mathfrak{C}}=<^{\mathfrak{A}} \cap\left(P^{\mathfrak{A}} \times P^{\mathfrak{A}}\right),
$$

is a continuous, dense linearly ordered set without endpoints. Suppose, by reduction to the absurd, that $P^{\mathfrak{A}}$ is countable. It follows, by Theorem 3.3, that $\mathfrak{C}$ is order-isomorphic to the structure of the rationals with order $\mathfrak{Q}=\left(Q,<^{\mathfrak{Q}}\right)$. But $\mathfrak{C}$ is continuous and $\mathfrak{Q}$ is not, which contradicts the fact that $\mathfrak{C}$ and $\mathfrak{Q}$ are order-isomorphic. It follows that $C=P^{\mathfrak{A}}$ is uncountable and so is $\mathfrak{A}$.

We immediately get:

Theorem 3.5 The Downward Löwenheim-Skolem Theorem for secondorder Circumscription does not hold.

We follow our investigation about the expressive power of Circumscription and we will show our second result of this section. We remember the definition of elementary and $\Delta$-elementary classes of models below (see, for instance, Ebbinghaus et al (1994)).

Definition 3.6 [Elementary and $\Delta$-Elementary Classes] Let $S$ be a symbol set and $\mathbb{C}$ a class of $S$-structures. $\mathbb{C}$ is said to be $\Delta$-elementary iff there is a set $T$ of $S$-sentences such that $\operatorname{Mod}_{S}(T)=\mathbb{C}$. $\mathbb{C}$ is said to be elementary if there is a single $S$-sentence $\phi$ such that $\operatorname{Mod}_{S}(\phi)=\mathbb{C}$.

From Theorem 3.5 it follows that there are some classes of minimal models of finite first-order theories which are not $\Delta$-elementary. An example is the sentence $T(P)$ in (3). For suppose that there is a set of first-order sentences $\Gamma$ whose models are exactly the same models of $\operatorname{Circ}_{S O}[T(P) ; P]$. Since the models of $\operatorname{Circ}_{S O}[T(P) ; P]$ are structures in the symbol set $\{<, P\}, \Gamma$ is a set of $\{<, P\}$-sentences. As firstorder sentences are finite, $\Gamma$ is countable. And since the Downward 
Löwenheim-Skolem Theorem does hold for first-order logic, $\Gamma$ has a countable or finite model, which contradicts Theorem 3.5.

Below, we will prove the main theorem of this section. We will show that there is no strictly $\Delta$-elementary class of $\bar{P} ; \bar{Z}$-minimal models of first-order sentences, that is, $\Delta$-elementary but not elementary. This will follow directly from the next theorem.

Theorem 3.7 Let $\phi(\bar{P} ; \bar{Z})$ be an $S^{\prime}=S \cup\{\bar{P}, \bar{Z}\}$-sentence such that the class $\mathbb{C}$ of $\bar{P} ; \bar{Z}$-minimal models of $\phi$ is $\Delta$-elementary. Then $\mathbb{C}$ is elementary.

Proof. In this proof, we will first define a first-order sentence $C_{\bar{P} ; \bar{Z}}^{\phi}$ and, after that, we will show that the models of $C_{\bar{P} ; \bar{Z}}^{\phi} \wedge \phi(\bar{P}, \bar{Z})$ are exactly the $\bar{P} ; \bar{Z}$-minimal models of $\phi(\bar{P}, \bar{Z})$. Let

$$
\Psi:=T h_{S^{\prime}}^{F O}(\mathbb{C})
$$

be the first-order $S^{\prime}$-theory of $\mathbb{C}$, that is, the set of all first-order sentences satisfied by all models in $\mathbb{C}$. As $\mathbb{C}$ is $\Delta$-elementary, then

$$
\operatorname{Mod}_{S^{\prime}}(\Psi)=\mathbb{C}
$$

Let $\overline{P^{\prime}}, \overline{Z^{\prime}}$ be new relation symbols, not occurring in $\phi(\bar{P}, \bar{Z})$, with the same arity of the predicate symbols $\bar{P}, \bar{Z}$, respectively. Let $\phi\left(\overline{P^{\prime}}, \overline{Z^{\prime}}\right)$ be the result of replacing $\bar{P}, \bar{Z}$ with $\overline{P^{\prime}}, \overline{Z^{\prime}}$ in $\phi(\bar{P}, \bar{Z})$. Consider the set

$$
\Psi \cup\left\{\phi\left(\overline{P^{\prime}}, \overline{Z^{\prime}}\right)\right\}
$$

of sentences. As neither some of $\overline{P^{\prime}}, \overline{Z^{\prime}}$ occurs in $\phi(\bar{P}, \bar{Z})$, nor some of $\bar{P}, \bar{Z}$ occurs in $\phi\left(\overline{P^{\prime}}, \overline{Z^{\prime}}\right)$, for each $S \cup\{\bar{P}, \bar{Z}\} \cup\left\{\overline{P^{\prime}}, \overline{Z^{\prime}}\right\}$-structure

$$
\mathfrak{C}:=\left(\mathfrak{A}, \overline{\mathbf{P}}, \overline{\mathbf{Z}}, \overline{\mathbf{P}^{\prime}}, \overline{\mathbf{Z}^{\prime}}\right)
$$


we have that

$$
\mathfrak{C}=\Psi \cup\left\{\phi\left(\overline{P^{\prime}}, \overline{Z^{\prime}}\right)\right\} \quad \text { iff } \quad(\mathfrak{A}, \overline{\mathbf{P}}, \overline{\mathbf{Z}}) \models \Psi \text { and }\left(\mathfrak{A}, \overline{\mathbf{P}^{\prime}}, \overline{\mathbf{Z}^{\prime}}\right) \models \phi\left(\overline{P^{\prime}}, \overline{Z^{\prime}}\right) \cdot(5)
$$

Consider the set

$$
\Psi \cup\left\{\phi\left(\overline{P^{\prime}}, \overline{Z^{\prime}}\right)\right\} \cup\left\{\bigvee_{1 \leq i \leq m} P_{i}^{\prime} \subsetneq P_{i}\right\}
$$

of sentences. Since the models of $\Psi$ are $\bar{P} ; \bar{Z}$-minimal, by (5) the set of sentences (6) is inconsistent. Let $\Theta^{\prime}$ be a finite subset of $\Psi \cup\left\{\phi\left(\overline{P^{\prime}}, \overline{Z^{\prime}}\right)\right\}$ such that, $\Theta^{\prime} \cup\left\{\bigvee_{1 \leq i \leq m} P_{i}^{\prime} \subsetneq P_{i}\right\}$ is inconsistent, and let $\Theta:=\Theta^{\prime}-$ $\phi\left(\overline{P^{\prime}}, \overline{Z^{\prime}}\right)$. As first-order logic is compact, such sets always exist. Let

$$
C_{\bar{P} ; \bar{Z}}^{\phi}:=\bigwedge \Theta
$$

be a first-order sentence obtained by the conjunction of all (finitely many) sentences in $\Theta$. By definition, any $\bar{P} ; \bar{Z}$-minimal model of $\phi(\bar{P}, \bar{Z})$ is a model of $\phi(\bar{P}, \bar{Z}) \wedge C_{\bar{P} ; \bar{Z}}^{\phi}$. Let $(\mathfrak{B}, \overline{\mathbf{P}}, \overline{\mathbf{Z}})$ be a model of $\phi(\bar{P}, \bar{Z}) \wedge$ $C_{\bar{P} ; \bar{Z}}^{\phi}$. Suppose $(\mathfrak{B}, \overline{\mathbf{P}}, \overline{\mathbf{Z}})$ is not a $\bar{P} ; \bar{Z}$-minimal model of $\phi(\bar{P}, \bar{Z})$. In this case, there is a model $\left(\mathfrak{B}, \overline{\mathbf{P}^{\prime}}, \overline{\mathbf{Z}^{\prime}}\right)$ of $\phi(\bar{P}, \bar{Z})$, such that $\left(\mathfrak{B}, \overline{\mathbf{P}^{\prime}}, \overline{\mathbf{Z}^{\prime}}\right)$ $\leq^{\bar{P}} ; \bar{Z}(\mathfrak{B}, \overline{\mathbf{P}}, \overline{\mathbf{Z}})$ and $\mathbf{P}_{i}^{\prime} \subsetneq \mathbf{P}_{i}$ for some $i, 1 \leq i \leq m$. It follows that

$$
\left(\mathfrak{B}, \overline{\mathbf{P}}, \overline{\mathbf{Z}}, \overline{\mathbf{P}^{\prime}}, \overline{\mathbf{Z}^{\prime}}\right) \models \Theta^{\prime} \cup\left\{\bigvee_{1 \leq i \leq m} P_{i}^{\prime} \subsetneq P_{i}\right\},
$$

which contradicts the fact that $\Theta^{\prime} \cup\left\{\bigvee_{1<i<m} P_{i}^{\prime} \subsetneq P_{i}\right\}$ is inconsistent. Therefore, an $S \cup\{\bar{P}, \bar{Z}\}$-structure is a $\bar{P} ; \bar{Z}$-minimal model of $\phi(\bar{P}, \bar{Z})$ iff it is a model of $\phi(\bar{P}, \bar{Z}) \wedge C_{\bar{P} ; \bar{Z}}^{\phi}$. That is, $\operatorname{Mod}_{S^{\prime}}\left(\phi(\bar{P}, \bar{Z}) \wedge C_{\bar{P} ; \bar{Z}}^{\phi}\right)$ is exactly the class of models $\mathbb{C}$. Hence, the class of $\bar{P} ; \bar{Z}$-minimal models of $\phi(\bar{P}, \bar{Z})$ is elementary. 
Corollary 3.8 Let $\phi(\bar{P} ; \bar{Z})$ be an $S^{\prime}=S \cup\{\bar{P}, \bar{Z}\}$-sentence such that

$$
\operatorname{Mod}_{S^{\prime}}\left(\operatorname{Circ}_{S O}[\phi(\bar{P} ; \bar{Z}) ; \bar{P} ; \bar{Z}]\right)
$$

is $\Delta$-elementary. Then $\operatorname{Mod}_{S^{\prime}}\left(\operatorname{Circ}_{S O}[\phi(\bar{P} ; \bar{Z}) ; \bar{P} ; \bar{Z}]\right)$ is elementary.

Theorem 3.5 shows that there are classes of minimal models of firstorder sentences which are not $\Delta$-elementary. Moreover, Theorem 3.7 shows that, if the class of minimal models of a first-order sentence is $\Delta$-elementary, then it is in fact elementary. The proof of the following corollary is immediate.

Corollary 3.9 The class of $\bar{P} ; \bar{Z}$-minimal models of a first-order formula is either elementary or it is not $\Delta$-elementary.

A particularly important consequence of Theorem 3.7 is:

Corollary 3.10 If a second-order circumscription is equivalent to a first-order theory, then the set of its conclusions is recursively enumerable.

In the following section, we will examine definability questions regarding first- and second-order Circumscription. We will begin with an explanation of the basic concepts of the Theory of Definitions. After that, we will give a detailed analysis of definability questions motivated by Doyle's work in Doyle (1985). We will apply the expressiveness results of this section to obtain the main results of Section 4 .

\section{DEFINABILITY AND CIRCUMSCRIPTION}

In Padoa (1900), Padoa argue that, in order to show that an expression like

$$
\forall \bar{x}(P(\bar{x}) \leftrightarrow \psi(\bar{x})),
$$

where the relation symbol $P$ does not occur in $\psi(\bar{x})$, cannot be proved from a theory, say, $\Gamma$, it is sufficient to show that there are two models 
of $\Gamma$ which differ on the interpretation of $P$, but agree on the interpretation of the other symbols. An expression like (7) is called an explicit definition. When an $S \cup\{P\}$-theory $\Gamma$ is such that for each two $S \cup\{P\}$ models $(\mathfrak{A}, \mathbf{P})$ and $\left(\mathfrak{A}, \mathbf{P}^{\prime}\right)$ of $\Gamma$ we always have $\mathbf{P}=\mathbf{P}^{\prime}$, we say that $\Gamma$ implicitly defines $P$.

The so called Padoa's Method consists of showing that an explicit definition for $P$ such as $\forall \bar{x}(P(\bar{x}) \leftrightarrow \psi(\bar{x}))$ cannot be a logical consequence of a set of first-order sentences, say $\Gamma$, by showing that $\Gamma$ does not implicitly defines $P$. This is equivalent to state that, if there is an explicit definition for $P$ in $\Gamma$, then $\Gamma$ implicitly defines $P$. In Beth (1953), Beth shows the converse. The Beth's Definability Theorem states that, if a set of first-order sentences $\Gamma$ implicitly defines $P$ in firstorder logic, then there is an explicit definition, say $\forall \bar{x}(P(\bar{x}) \leftrightarrow \psi(\bar{x}))$ in first-order logic for $P$ such that

$$
\Gamma \models \forall \bar{x}(P(\bar{x}) \leftrightarrow \psi(\bar{x}))
$$

In Doyle (1985), Doyle investigated some relations between Circumscription and implicit definability in the context of first-order Circumscription. Doyle addressed the question on what are the circumstances in which first-order Circumscription implicitly defines the circumscribed relation. It is intuitive that such circumstances must involve the existence of exactly one minimal interpretation for the circumscribed relation in the domain of a structure, that is, given a symbol set $S^{\prime}=S \cup\{P, \bar{Z}\}$ and a sentence $\phi(P, \bar{Z})$, for each $S$-structure $\mathfrak{A}$, there is at most one $P^{\mathfrak{A}} \subset A$ such that $\mathfrak{B}=\left(\mathfrak{A}, P^{\mathfrak{A}}, \bar{Z}^{\mathfrak{A}}\right)$ is a $P ; \bar{Z}$ minimal model of $\phi(P, \bar{Z})$ for some (possibly many) $\bar{Z}^{\mathfrak{A}}$. This semantic condition, stated in another, equivalent form, was called the unique minimal model property by Moinard and Rolland in Moinard, Rolland (1991), who also introduced a variant of the standard Circumscription called Strong Circumscription Moinard, Rolland (1991) and gave some 
sufficient conditions for the equivalence between standard and Strong Circumscription. Here, we consider another, different point addressed by Doyle in Doyle (1985) relating Circumscription with Definability Theory, which we explain below.

Suppose $\operatorname{Circ}_{F O}[\phi ; P ; \bar{Z}]$ implicitly defines $P$. By Beth's Definability Theorem, there is an explicit definition $\forall \bar{x}(P(\bar{x}) \leftrightarrow \psi(\bar{x}))$ for $P$. In Doyle (1985), Doyle states that it is not always the case that the circumscription $\operatorname{Circ}_{F O}[\phi ; P ; \bar{Z}]$ of $P$ in $\phi$ can be replaced with $\phi$ plus its explicit definition $\forall \bar{x}(P(\bar{x}) \leftrightarrow \psi(\bar{x}))$. In other words, it may be the case that, for some $\phi$,

$$
\operatorname{Circ}_{F O}[\phi ; P ; \bar{Z}] \not \equiv \phi \wedge \forall \bar{x}(P(\bar{x}) \leftrightarrow \psi(\bar{x}))
$$

That is, an explicit definition for the implicitly defined relation $P$ cannot replace the (infinitely many) sentences in $\operatorname{Circ}_{F O}[\phi ; P ; \bar{Z}]$. However, Doyle's example fails to show this. Doyle considered the sentence $\phi(B l o c k)$ of block's world:

$$
\phi(B l o c k):=\operatorname{Block}\left(t_{1}\right) \wedge \ldots \wedge \operatorname{Block}\left(t_{l}\right) .
$$

It is clear that for any model $\mathfrak{A}$ of $\phi(B l o c k), t_{i}^{\mathfrak{A}}$ must be included in $B l o c k^{\mathfrak{A}}$, where Block $k^{\mathfrak{A}}$ is the interpretation of the relation symbol Block by $\mathfrak{A}$. It follows that the Block-minimal models of $\phi(B l o c k)$ are those in which

$$
\text { Block }^{\mathfrak{A}}=\left\{t_{1}^{\mathfrak{A}}, \ldots, t_{l}^{\mathfrak{A}}\right\} .
$$

For each Block-minimal model $\mathfrak{B}$ of $\phi(B l o c k)$, we have that

$$
\mathfrak{B} \models \forall x\left(\operatorname{Block}(x) \leftrightarrow\left(x=t_{1} \vee \ldots \vee x=t_{l}\right)\right),
$$

and for $\psi(x):=\left(x=t_{1} \vee \ldots \vee x=t_{l}\right)$, the instance

$$
\phi(\psi(x)) \wedge \forall x(\psi(x) \rightarrow \operatorname{Block}(x)) \rightarrow \forall x(\operatorname{Block}(x) \rightarrow \psi(x))
$$


of the circumscription schema for Block in $\phi(B l o c k)$ - see the schema (1)-belongs to $\operatorname{Circ}_{F O}[\phi(B l o c k) ; B l o c k]$. This means that the Blockminimal models of $\phi(B l o c k)$ are exactly the models of $\operatorname{Circ}_{F O}[\phi(B l o$ $c k) ; B l o c k]$. It can be easily seen that

$$
\operatorname{Circ}_{F O}[\phi(B l o c k) ; B l o c k] \equiv \phi(B l o c k) \wedge \forall x(B \operatorname{Block}(x) \leftrightarrow \psi(x))
$$

contrary to what was expected by Doyle in Doyle (1985, p. 397), where he supposed to be necessary additional axioms to guarantee minimality.

In the following, we will carefully investigate this question for both first- and second-order Circumscription.

There is a wide class of sentences for which (8) fails. First, let us precisely state the concept of well-foundedness for sentences in the context of minimal models.

Definition 4.1 [Well-Founded Sentences] We say that a sentence $\phi(\bar{P}, \bar{Z})$ is $\bar{P} ; \bar{Z}$-well-founded iff for each model $\mathfrak{A}$ of $\phi(\bar{P}, \bar{Z})$ there is a $\bar{P} ; \bar{Z}$-minimal model $\mathfrak{B}$ of $\phi(\bar{P}, \bar{Z})$ such that $\mathfrak{B} \leq \bar{P} ; \bar{Z} \mathfrak{A}$. If $\bar{Z}=\emptyset$ we write $\bar{P}$-well-founded for $\bar{P} ; \emptyset$-well-founded.

For $\bar{P}$-well-founded sentences in circumscriptions without varied relations, (8) does not hold.

Theorem 4.2 Let the $S^{\prime}$-sentence $\phi(P)$ be a P-well-founded sentence and $\mathbb{C}$ the class of P-minimal models of $\phi(P)$. Suppose that $\mathbb{C}$ is $P$ defined and that $\forall \bar{x}(P(\bar{x}) \leftrightarrow \psi(\bar{x}))$ is a first-order explicit definition for $P$ in $\mathbb{C}$, which means that $\phi(\bar{P}, \bar{Z}) \models \bar{P} ; \bar{Z} \forall \bar{x}(P(\bar{x}) \leftrightarrow \psi(\bar{x}))$. Then

$$
\mathbb{C}=\operatorname{Mod}_{S^{\prime}}(\phi(P) \wedge \forall \bar{x}(P(\bar{x}) \leftrightarrow \psi(\bar{x})))
$$

Proof. By the hypothesis of the theorem,

$$
\mathbb{C} \subseteq \operatorname{Mod}_{S^{\prime}}(\phi(P) \wedge \forall \bar{x}(P(\bar{x}) \leftrightarrow \psi(\bar{x})))
$$


Suppose by contradiction that there is a model

$$
\mathfrak{A} \in \operatorname{Mod}_{S^{\prime}}(\phi(P) \wedge \forall \bar{x}(P(\bar{x}) \leftrightarrow \psi(\bar{x})))
$$

which is not a $P$-minimal model of $\phi(P)$. As $\phi(P)$ is $P$-well-founded, there is a $P$-minimal model $\mathfrak{B}$ of $\phi(P)$ such that $\mathfrak{B} \leq^{P} \mathfrak{A}$. By $(9)$,

$$
\mathfrak{B} \models \forall \bar{x}(P(\bar{x}) \leftrightarrow \psi(\bar{x}))
$$

But since $\mathfrak{B} \leq^{P} \mathfrak{A}, \mathfrak{A}$ and $\mathfrak{B}$ agree on the interpretation of the symbols in $S^{\prime}-\{P\}$. Since $\mathfrak{A}$ and $\mathfrak{B}$ are models of $\forall \bar{x}(P(\bar{x}) \leftrightarrow \psi(\bar{x}))$, it follows that $\mathfrak{A}$ and $\mathfrak{B}$ agree on $P$ and, hence, $\mathfrak{A}=\mathfrak{B}$. Thus

$$
\operatorname{Mod}_{S^{\prime}}(\phi(P) \wedge \forall \bar{x}(P(\bar{x}) \leftrightarrow \psi(\bar{x}))) \subseteq \mathbb{C} .
$$

Corollary 4.3 Let $\phi(P)$ be a P-well-founded sentence.

1. If $\operatorname{Circ}_{F O}[\phi(P) ; P]$ implicitly defines $P$ and $\forall \bar{x}(P(\bar{x}) \leftrightarrow \psi(\bar{x}))$ is a first-order explicit definition for $P$ in $\operatorname{Circ}_{F O}[\phi(P) ; P]$, then

$$
\operatorname{Circ}_{F O}[\phi(P) ; P] \equiv \phi(P) \wedge \forall \bar{x}(P(\bar{x}) \leftrightarrow \psi(\bar{x}))
$$

2. If $\operatorname{Circ}_{S O}[\phi(P) ; P]$ implicitly defines $P, \forall \bar{x}(P(\bar{x}) \leftrightarrow \psi(\bar{x}))$ is a first-order explicit definition for $P$ in $\operatorname{Circ}_{S O}[\phi(P) ; P]$, then

$$
\operatorname{Circ}_{S O}[\phi(P) ; P] \equiv \phi(P) \wedge \forall \bar{x}(P(\bar{x}) \leftrightarrow \psi(\bar{x})) .
$$

Proof. The proof of 2 follows directly from Theorem 4.2. The proof of 1 is analogous, since any $P$-minimal model of $\phi(P)$ is a model of $\operatorname{Circ}_{F O}[\phi(P) ; P]$.

Although Doyle's example does not satisfy the statement (8), there are sentences $\phi$ for which that statement is true. Indeed, there are well- 
founded sentences which satisfy the statement (8) for circumscription with varied predicates. We show this in the following example, where we use second-order Circumscription since, in this case, it is equivalent to first-order Circumscription.

Example 4.4 Let $P$ and $Z$ be unary relations. Consider the sentence

$$
\phi(P, Z):=\forall x(\neg P(x) \rightarrow Z(x))
$$

For each model $\mathfrak{B}$ of $\phi(P, Z)$, there is a $P ; Z$-minimal model $\mathfrak{B}^{\prime}$ such that $\mathfrak{B}^{\prime} \leq P ; Z \mathfrak{B}$, namely with $P^{\mathfrak{B}^{\prime}}=\emptyset, Z^{\mathfrak{B}^{\prime}}=B$ and $s^{\mathfrak{B}^{\prime}}=s^{\mathfrak{B}}$ for the other symbols $s$ in the symbol set. Hence, $\phi(P, Z)$ is $P ; Z$-wellfounded. Moreover, any $P ; Z$-minimal model of $\phi(P, Z)$ interprets $P$ as the empty relation. Consider the explicit definition $\forall x(P(x) \leftrightarrow \neg Z(x))$ for $P$. It is clear that

$$
\operatorname{Circ}_{S O}[\phi(P, Z) ; P ; Z] \models \forall x(P(x) \leftrightarrow \neg Z(x)),
$$

but

$$
\operatorname{Circ}_{S O}[\phi(P, Z) ; P ; Z] \not \equiv \phi(P, Z) \wedge \forall x(P(x) \leftrightarrow \neg Z(x))
$$

Based on the proof of Theorem 4.2, we can give a restricted version of this theorem for well-founded sentences in parallel circumscriptions with varied relations.

Theorem 4.5 Let the $S^{\prime}$-sentence $\phi(\bar{P}, \bar{Z})$ be a $\bar{P} ; \bar{Z}$-well-founded sentence and $\mathbb{C}$ the class of $\bar{P} ; \bar{Z}$-minimal models of $\phi(\bar{P}, \bar{Z})$. Suppose that $\mathbb{C}$ is $P_{i}$-defined and that $\forall \bar{x}_{i}\left(P_{i}\left(\bar{x}_{i}\right) \leftrightarrow \psi_{i}\left(\bar{x}_{i}\right)\right)$ is a first-order explicit definition for $P_{i}$ in $\mathbb{C}$, for each $P_{i} \in \bar{P}$, that is, $\phi(\bar{P}, \bar{Z}) \models \bar{P} ; \bar{Z}$ $\forall \bar{x}_{i}\left(P_{i}\left(\bar{x}_{i}\right) \leftrightarrow \psi_{i}\left(\bar{x}_{i}\right)\right)$, and such that no symbol in $\{\bar{P}, \bar{Z}\}$ occurs in $\psi_{i}$, for each $P_{i} \in \bar{P}$. Then

$$
\mathbb{C}=\operatorname{Mod}_{S^{\prime}}\left(\phi(\bar{P}, \bar{Z}) \wedge \bigwedge_{P_{i} \in \bar{P}} \forall \bar{x}_{i}\left(P_{i}\left(\bar{x}_{i}\right) \leftrightarrow \psi_{i}\left(\bar{x}_{i}\right)\right)\right)
$$

Manuscrito - Rev. Int. Fil., Campinas, v. 34, n. 1, p. 233-266, jan.-jun. 2011. 
Proof. Analogous to the proof of Theorem 4.2.

Corollary 4.6 Let $\phi(\bar{P}, \bar{Z})$ be a $\bar{P} ; \bar{Z}$-well-founded sentence.

1. If $\operatorname{Circ}_{F O}[\phi(\bar{P}, \bar{Z}) ; \bar{P} ; \bar{Z}]$ implicitly defines $P_{i}$ and $\forall \bar{x}_{i}\left(P_{i}\left(\bar{x}_{i}\right) \leftrightarrow\right.$ $\left.\psi_{i}\left(\bar{x}_{i}\right)\right)$ is a first-order explicit definition for $P_{i}$ in $\operatorname{Circ}_{F O}[\phi(\bar{P}, \bar{Z})$; $\bar{P} ; \bar{Z}]$ such that no symbol in $\bar{P}, \bar{Z}$ occurs in $\psi_{i}(\bar{x})$, for each $P_{i} \in \bar{P}$, then

$$
\operatorname{Circ}_{F O}[\phi(\bar{P}, \bar{Z}) ; \bar{P} ; \bar{Z}] \equiv \phi(\bar{P}, \bar{Z}) \wedge \bigwedge_{P_{i} \in \bar{P}} \forall \bar{x}_{i}\left(P_{i}\left(\bar{x}_{i}\right) \leftrightarrow \psi_{i}\left(\bar{x}_{i}\right)\right)
$$

2. If $\operatorname{Circ}_{S O}[\phi(\bar{P}, \bar{Z}) ; \bar{P} ; \bar{Z}]$ implicitly defines $P_{i}, \forall \bar{x}_{i}\left(P_{i}\left(\bar{x}_{i}\right) \leftrightarrow \psi\left(\bar{x}_{i}\right)\right)$ is a first-order explicit definition for $P_{i}$ in $\operatorname{Circ}_{S O}[\phi(\bar{P}, \bar{Z})$; $\bar{P} ; \bar{Z}]$ such that no symbol in $\bar{P}, \bar{Z}$ occurs in $\psi_{i}\left(\bar{x}_{i}\right)$, for each $P_{i} \in \bar{P}$, then

$$
\operatorname{Circ}_{S O}[\phi(\bar{P}, \bar{Z}) ; \bar{P} ; \bar{Z}] \equiv \phi(\bar{P}, \bar{Z}) \wedge \bigwedge_{P_{i} \in \bar{P}} \forall \bar{x}_{i}\left(P_{i}\left(\bar{x}_{i}\right) \leftrightarrow \psi_{i}\left(\bar{x}_{i}\right)\right)
$$

Proof. Analogous to the proof of Corollary 4.3.

The restriction on the occurrence of varied relations in the explicit definition used in Corollary 4.6 does not work for non-well-founded sentences, since there are non-well-founded sentences for which (8) does hold even under this restriction. Again, in the following example, firstand second-order Circumscription coincide.

Example 4.7 Suppose < be a linear order relation. Let

$$
\begin{gathered}
L E(x):=\forall y(x<y \vee x=y) \text { and } \\
G E(x):=\forall y(y<x \vee y=x)
\end{gathered}
$$


be first-order formulas saying that $x$ is the least (LE) and the greatest $(G E)$ element of $<$, respectively, and

$$
S(x, y):=x<y \wedge \forall z(x<z \rightarrow y<z \vee z=y)
$$

be a first-order formula saying that $y$ is the successor of $x$ with respect to $<$. Let $\alpha(<)$ be a first-order sentence which says that $<$ is a linear order (a total, transitive and asymmetric binary relation) in which each element, except the greatest, has a successor and each element, except the least, has a predecessor, although $\alpha(<)$ does not determine whether there are such least and greatest elements or not:

$$
\begin{aligned}
\alpha(<):= & \forall x \forall y(x<y \rightarrow \neg y<x) \wedge \forall x \forall y \forall z(x<y \wedge y<z \rightarrow x<z) \wedge \\
& \forall x \forall y(x<y \vee x=y \vee y<x) \wedge \forall x(\exists y(S(x, y)) \leftrightarrow \neg G E(x)) \wedge \\
& \forall x(\exists y(S(y, x)) \leftrightarrow \neg L E(x)) .
\end{aligned}
$$

Let $c$ be a constant symbol in the underlying symbol set $S^{\prime}$. Let $\beta(c)$ be a first-order formula expressing that, if $<$ has a least element, then c is such least element:

$$
\beta(c):=\exists x(L E(x)) \rightarrow L E(c) .
$$

Let $P$ be a relation symbol in $S^{\prime}$. Let $\gamma(P)$ be a first-order sentence saying that $P$ is not empty, $P$ has a greatest element with respect to $<$ and $P$ is downward closed, that is, if a belongs to $P$ and $b<a$, then $b$ belongs to $P$ :

$$
\gamma(P):=N E(P) \wedge \exists x(P(x) \wedge(\forall y(P(y) \rightarrow y<x \vee y=x))) \wedge D C(P) .
$$

It is clear that $<$ has a least element in any $P$-minimal model $(\mathfrak{A}, \mathbf{P})$ of

$$
\alpha(<) \wedge \beta(c) \wedge \gamma(P)
$$

Manuscrito - Rev. Int. Fil., Campinas, v. 34, n. 1, p. 233-266, jan.-jun. 2011. 
otherwise any downward closed proper subset $\mathbf{P}^{\prime}$ of $\mathbf{P}$ could be used to construct a model $\left(\mathfrak{A}, \mathbf{P}^{\prime}\right) \leq{ }^{P}(\mathfrak{A}, \mathbf{P})$. Conversely, if $<$ has a least element, then $\alpha(<) \wedge \beta(c) \wedge \gamma(P)$ has a P-minimal model in which $P^{\mathfrak{A}}=\{c\}$. We can see that $\operatorname{Circ}_{S O}[\alpha(<) \wedge \beta(c) \wedge \gamma(P) ; P]$ is equivalent to the set $\Gamma$ of first-order formulas defined as

$$
\Gamma:=\{\alpha(<) \wedge \beta(c) \wedge \gamma(P), \forall x(P(x) \rightarrow L E(x))\}
$$

It can easily be seen that the formula $\forall x(P(x) \leftrightarrow(x=c \vee x<c))$ is an explicit definition for $P$ in $\Gamma$, that is

$$
\Gamma \models \forall x(P(x) \leftrightarrow(x=c \vee x<c)) .
$$

However, as $\alpha(<) \wedge \beta(c) \wedge \gamma(P)$ has models (not minimal) in which $<$ does not have a least element, we have that

$$
\begin{gathered}
\operatorname{Circ}_{S O}[\alpha(<) \wedge \beta(c) \wedge \gamma(P) ; P] \\
\not \equiv \\
\alpha(<) \wedge \beta(c) \wedge \gamma(P) \wedge \forall x(P(x) \leftrightarrow(x=c \vee x<c)) .
\end{gathered}
$$

So, if, in (8), $\phi$ is the sentence $\alpha(<) \wedge \beta(c) \wedge \gamma(P)$ and $\psi$ is $L E(x)$, then (8) is verified.

It is important to stress the fact that, for a relation implicitly defined in a theory, there are many distinct explicit definitions. If we consider the explicit definition $\forall x(P(x) \leftrightarrow(x=c))$ for $P$ and $\Gamma$ as in Example 4.7, we have 


$$
\Gamma \models \forall x(P(x) \leftrightarrow(x=c)),
$$

and

$\operatorname{Circ}_{S O}[\alpha(<) \wedge \beta(c) \wedge \gamma(P) ; P] \equiv \alpha(<) \wedge \beta(c) \wedge \gamma(P) \wedge \forall x(P(x) \leftrightarrow(x=c))$.

That is, if $\psi$ is $(x=c)$ in (8), that statement is not verified.

Note that the problem in Example 4.7 occurs in the cases in which $<$ does not have a least element. In such cases, there is no interpretation for $P$ which gives a minimal model for $\alpha(<) \wedge \beta(c) \wedge \gamma(P)$. It follows that $\alpha(<) \wedge \beta(c) \wedge \gamma(P)$ is not $P$-well-founded, falling outside the scope of Corollary 4.3 or Corollary 4.6.

In the face of these facts, we can ask: in the cases in which $\operatorname{Circ}_{S O}[\phi ; P ; \bar{Z}]$ implicitly defines $P$ and $\operatorname{Mod}_{S^{\prime}}\left(\operatorname{Circ}_{S O}[\phi ; P ; \bar{Z}]\right)$ is $\Delta$ elementary, is there an explicit definition $\forall \bar{x}(P(\bar{x}) \leftrightarrow \psi(\bar{x}))$ for which

$$
\operatorname{Circ}_{S O}[\phi ; P ; \bar{Z}] \equiv \phi \wedge \forall \bar{x}(P(\bar{x}) \leftrightarrow \psi(\bar{x}))
$$

holds? We will give a positive answer to this question below.

Theorem 4.8 Let $\mathbb{C}$ be the class of $\bar{P} ; \bar{Z}$-minimal models of the $S^{\prime}$ sentence $\phi(\bar{P}, \bar{Z})$ and such that $\mathbb{C}$ is $\Delta$-elementary and $P_{i}$-defined for some $i, 1 \leq i \leq m$. Then there is an explicit definition $\forall \bar{x}\left(P_{i}(\bar{x}) \leftrightarrow\right.$ $\psi(\bar{x}))$ such that

$$
\mathbb{C}=\operatorname{Mod}_{S^{\prime}}\left(\phi(\bar{P}, \bar{Z}) \wedge \forall \bar{x}\left(P_{i}(\bar{x}) \leftrightarrow \psi(\bar{x})\right)\right)
$$

Proof. Let $\phi(\bar{P}, \bar{Z})$ be an $S^{\prime}=S \cup\{\bar{P}, \bar{Z}\}$ sentence of first-order logic, $P_{i}$ be an $n$-ary relation symbol and $\bar{P}$ and $\bar{Z}$ tuples of relation symbols. By Theorem 3.7, there is a sentence $\gamma(\bar{P}, \bar{Z})=C_{\bar{P} ; \bar{Z}}^{\phi} \wedge \phi(\bar{P}, \bar{Z})$ such that

$$
\mathbb{C}=\operatorname{Mod}_{S^{\prime}}(\gamma(\bar{P}, \bar{Z}))
$$


As $\mathbb{C}$ is $P_{i}$-defined, by Beth's Theorem there is an explicit definition $\forall \bar{x}\left(P_{i}(\bar{x}) \leftrightarrow \psi(\bar{x})\right)$ for which

$$
\gamma(\bar{P}, \bar{Z}) \models \forall \bar{x}\left(P_{i}(\bar{x}) \leftrightarrow \psi(\bar{x})\right)
$$

Let $\gamma^{\prime}:=\gamma(\psi, \bar{Z})$ be the $S^{\prime}-\left\{P_{i}\right\}$-sentence obtained from $\gamma(\bar{P}, \bar{Z})$ by replacing each atom $P_{i}(\bar{t})$ with $\psi(\bar{t})$ in $\gamma(\bar{P}, \bar{Z})$, with the appropriated renaming of the bound variable of $\psi(\bar{t})$ in order to avoid undesirable binding of the variables in $\bar{t}$. Let the $S^{\prime}-\left\{P_{i}\right\}$-structure $\mathfrak{A}$ be a model of $\gamma^{\prime}$ and $\mathbf{P}_{i} \subseteq A^{n}$ defined as $\mathbf{P}_{i}:=\left\{\bar{a} \in A^{n} \mid \mathfrak{A} \models \psi(\bar{x})[\bar{a}]\right\}$. Then, by (11), $\left(\mathfrak{A}, \mathbf{P}_{i}\right) \models \gamma(\bar{P}, \bar{Z})$. On the other hand, again by (11), if $\left(\mathfrak{A}, \mathbf{P}_{i}\right) \models \gamma(\bar{P}, \bar{Z})$ then $\mathfrak{A} \models \gamma^{\prime}$ and $\mathbf{P}_{i}:=\left\{\bar{a} \in A^{n} \mid \mathfrak{A} \models \psi(\bar{x})[\bar{a}]\right\}$. It follows that

$$
\left(\mathfrak{A}, \mathbf{P}_{i}\right) \models \gamma(\bar{P}, \bar{Z}) \quad \text { iff } \quad \mathfrak{A} \models \gamma^{\prime} \text { and } \mathbf{P}_{i}=\left\{\bar{a} \in A^{n} \mid \mathfrak{A} \models \psi(\bar{x})[\bar{a}]\right\}(12)
$$

Let $\psi^{\prime}(\bar{x}):=\gamma^{\prime} \wedge \psi(\bar{x})$. Let $\left(\mathfrak{A}, \mathbf{P}_{i}\right)$ be a model of $\gamma(\bar{P}, \bar{Z})$, where $\mathfrak{A}$ is an $S^{\prime}-\left\{P_{i}\right\}$-structure. Since $\gamma(\bar{P}, \bar{Z})=C_{\bar{P} ; \bar{Z}}^{\phi} \wedge \phi$, we have that

$$
\left(\mathfrak{A}, \mathbf{P}_{i}\right) \models \phi(\bar{P}, \bar{Z}) .
$$

By (11), it follows that $\left(\mathfrak{A}, \mathbf{P}_{i}\right) \models \forall \bar{x}\left(P_{i}(\bar{x}) \leftrightarrow \psi(\bar{x})\right)$. Let $\overline{\mathbf{a}} \in A^{n}$ be a tuple of elements in $A$. We have that

$$
\left(\mathfrak{A}, \mathbf{P}_{i}\right) \models P_{i}(\bar{x})[\overline{\mathbf{a}}] \text { iff }\left(\mathfrak{A}, \mathbf{P}_{i}\right) \models \psi(\bar{x})[\overline{\mathbf{a}}],
$$

and, by (12),

$$
\left(\mathfrak{A}, \mathbf{P}_{i}\right) \models \psi(\bar{x})[\overline{\mathbf{a}}] \text { iff }\left(\mathfrak{A}, \mathbf{P}_{i}\right) \models \gamma^{\prime} \wedge \psi(\bar{x})[\overline{\mathbf{a}}] .
$$

From (14) and (15), we get

$$
\left(\mathfrak{A}, \mathbf{P}_{i}\right) \models \forall \bar{x}\left(P_{i}(\bar{x}) \leftrightarrow \psi^{\prime}(\bar{x})\right) .
$$


By (13) and (16),we have

$$
\left(\mathfrak{A}, \mathbf{P}_{i}\right) \models \phi(\bar{P}, \bar{Z}) \wedge \forall \bar{x}\left(P_{i}(\bar{x}) \leftrightarrow \psi^{\prime}(\bar{x})\right)
$$

As (17) holds for any model $\left(\mathfrak{A}, \mathbf{P}_{i}\right)$ of $\gamma(\bar{P}, \bar{Z})$, it follows that

$$
\gamma(\bar{P}, \bar{Z}) \models \phi(\bar{P}, \bar{Z}) \wedge \forall \bar{x}\left(P_{i}(\bar{x}) \leftrightarrow \psi^{\prime}(\bar{x})\right) .
$$

Now, let $\left(\mathfrak{A}, \mathbf{P}_{i}\right)$ be a model of $\phi(\bar{P}, \bar{Z}) \wedge \forall \bar{x}\left(P_{i}(\bar{x}) \leftrightarrow \psi^{\prime}(\bar{x})\right)$. If $\mathbf{P}_{i}=\emptyset$, then $\left(\mathfrak{A}, \mathbf{P}_{i}\right)$ is a $\bar{P} ; \bar{Z}$-minimal model of $\phi(\bar{P}, \bar{Z})$. If $\mathbf{P}_{i} \neq \emptyset$, then there is $\bar{a}$ in $A^{n}$ such that $\left(\mathfrak{A}, \mathbf{P}_{i}\right) \models P_{i}(\bar{x})[\bar{a}]$. But, as $\left(\mathfrak{A}, \mathbf{P}_{i}\right) \models$ $\forall \bar{x}\left(P_{i}(\bar{x}) \leftrightarrow \psi^{\prime}(\bar{x})\right)$, then $\left(\mathfrak{A}, \mathbf{P}_{i}\right) \models \psi^{\prime}(\bar{x})[\bar{a}]$ and hence $\left(\mathfrak{A}, \mathbf{P}_{i}\right) \models \gamma^{\prime} \wedge$ $\psi(\bar{x})[\bar{a}]$. Therefore $\left(\mathfrak{A}, \mathbf{P}_{i}\right) \models \gamma^{\prime}$, and, as $P_{i}$ does not occur in $\gamma^{\prime}$,

$$
\mathfrak{A} \models \gamma^{\prime} .
$$

Since $\left(\mathfrak{A}, \mathbf{P}_{i}\right) \models \forall \bar{x}\left(P_{i}(\bar{x}) \leftrightarrow \psi^{\prime}(\bar{x})\right)$, we have that $\mathbf{P}_{i}=\left\{\bar{a} \in A^{n} \mid \mathfrak{A} \models\right.$ $\left.\psi^{\prime}(\bar{x})[\bar{a}]\right\}$. But as $\mathfrak{A} \models \gamma^{\prime}$, it follows that

$$
\mathbf{P}_{i}=\left\{\bar{a} \in A^{n} \mid \mathfrak{A} \models \psi^{\prime}(\bar{x})[\bar{a}]\right\}=\left\{\bar{a} \in A^{n} \mid \mathfrak{A} \models \psi(\bar{x})[\bar{a}]\right\} .
$$

From (12), (19) and (20), we have

$$
\left(\mathfrak{A}, \mathbf{P}_{i}\right) \models \gamma(\bar{P}, \bar{Z}),
$$

and hence

$$
\phi(\bar{P}, \bar{Z}) \wedge \forall \bar{x}\left(P_{i}(\bar{x}) \leftrightarrow \psi^{\prime}(\bar{x})\right) \models \gamma(\bar{P}, \bar{Z}) .
$$

By (18) and (21) we have $\mathbb{C}=\operatorname{Mod}_{S^{\prime}}(\gamma(\bar{P}, \bar{Z}))=\operatorname{Mod}_{S^{\prime}}(\phi(\bar{P}, \bar{Z}) \wedge$ $\left.\forall \bar{x}\left(P_{i}(\bar{x}) \leftrightarrow \psi^{\prime}(\bar{x})\right)\right)$.

From the proof of the Theorem 4.8 above, we have the following: 
Corollary 4.9 Let $\mathbb{C}$ be the class of $\bar{P} ; \bar{Z}$-minimal models of the $S^{\prime}$-sentence $\phi(\bar{P}, \bar{Z})$ and such that $\mathbb{C}$ is $\Delta$-elementary and there is $J \subseteq\{1, \ldots, m\}$ such that $\mathbb{C}$ is $P_{i}$-defined for all $i \in J$. Then there are explicit definitions $\forall \bar{x}\left(P_{i}\left(\bar{x}_{i}\right) \leftrightarrow \psi_{i}\left(\bar{x}_{i}\right)\right), i \in J$, such that $\mathbb{C}=$ $\operatorname{Mod}_{S^{\prime}}\left(\phi(\bar{P}, \bar{Z}) \wedge \bigwedge_{i \in J} \forall \bar{x}_{i}\left(P_{i}\left(\bar{x}_{i}\right) \leftrightarrow \psi_{i}\left(\bar{x}_{i}\right)\right)\right)$.

Corollary 4.10 If $\operatorname{Circ}_{S O}[\phi(\bar{P}, \bar{Z}) ; \bar{P} ; \bar{Z}]$ implicitly defines $P_{i}, i \in J$ for some $J \in\{1, \ldots, m\}$ and $\operatorname{Mod}_{S^{\prime}}\left(\operatorname{Circ}_{S O}[\phi(\bar{P}, \bar{Z}) ; \bar{P} ; \bar{Z}]\right)$ is $\Delta$ elementary, then there are explicit definitions $\forall \bar{x}_{i}\left(P_{i}\left(\bar{x}_{i}\right) \leftrightarrow \psi\left(\bar{x}_{i}\right)\right)$ such that

$$
\operatorname{Circ}_{S O}[\phi(\bar{P}, \bar{Z}) ; \bar{P} ; \bar{Z}] \equiv \phi(\bar{P}, \bar{Z}) \wedge \bigwedge_{i \in J} \forall \bar{x}_{i}\left(P_{i}\left(\bar{x}_{i}\right) \leftrightarrow \psi_{i}\left(\bar{x}_{i}\right)\right)
$$

\section{CONCLUSIONS}

We investigated expressiveness and definability results that concerns Circumscription. We dealt with both first- and second-order Circumscription. Within first-order Circumscription, the result of circumscribing a relation $P$ with varied $\bar{Z}$ is a set of first-order sentences, namely the set of all first-order instances of the circumscription schema (1), see Section 3. Second-order Circumscription results in a secondorder sentence whose models are just the minimal models of the circumscribed sentence. In Section 3, Theorem 3.5, we gave a proof that second-order Circumscription does not have the Löwenheim-Skolem Theorem. We showed that second-order Circumscription can express a class of structures which has a dense, continuous, linearly-ordered set without end-points as a substructure. Thus, any model in such class is uncountable. In Theorem 3.7 we showed that, whenever the class of minimal models of a first-order sentence is $\Delta$-elementary, such class is actually elementary. That is, whenever a second-order circumscription is equivalent to a first-order theory, this theory is finitely axiomatizable. 
As stressed by Corollary 3.8, Theorem 3.5 and Theorem 3.7 divide the classes of minimal models of first-order sentences in elementary and non- $\Delta$-elementary. In a picture, we have:

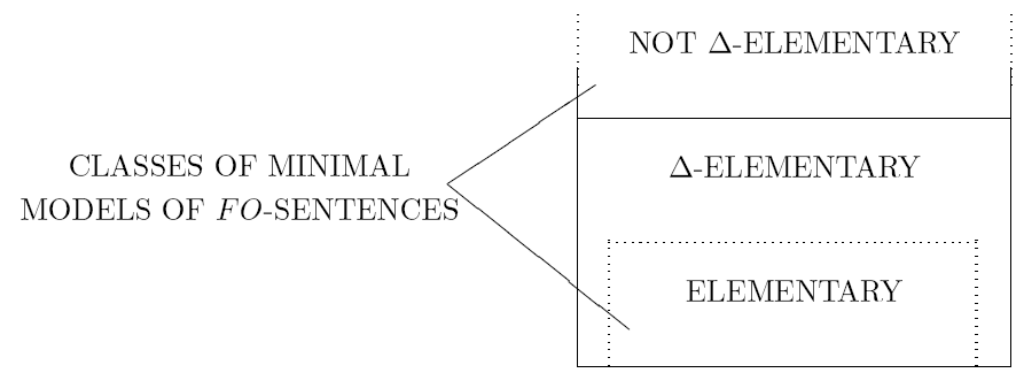

Figure 1: Classes of minimal models of first-order sentences.

A straightforward but important consequence is that, when the class of minimal models of a first-order sentence is $\Delta$-elementary, the set of logical consequences of the circumscribed theory is recursively enumerable, as pointed out in Corollary 3.10.

We also investigated definability questions regarding Circumscription. In Moinard, Rolland (1991), Moinard and Rolland dealt with a problem raised by Doyle in Doyle (1985) about the conditions on which Circumscription defines the circumscribed relation. We dealt with another, although related, problem here. We are concerned with a statement made by Doyle also in Doyle (1985). Doyle stated that, in the cases in which a first-order circumscription implicitly defines the circumscribed relation, such circumscription cannot always be replaced by the initial sentence together with an explicit definition for the circumscribed relation. He also argued that, in some cases, extra axioms should be added in order to guarantee minimality Doyle (1985, p. 397). Unfortunately, Doyle's example fails in showing this (see Sec- 
tion 4 above). Indeed, although Doyle's example does not work, he was right about the fact that the circumscription of a relation cannot always be replaced by the initial theory and an explicit definition for the circumscribed relation. Examples 4.4 and 4.7 in Section 4 confirm his statement. However, there is a wide class of first-order sentences for which his statement does not hold, namely the $P$-well-founded sentences, as showed by Theorem 4.2. We called attention to the fact that an implicitly defined symbol can have many different explicit definitions. We then asked about the existence of a suitable explicit definition $\psi$ which yields the equivalence between the circumscription and the initial theory plus $\psi$. In Corollary 4.10, we showed that, whenever the first-order logic can express the minimality of a relation in a sentence with varied relations, if the circumscription of a relation implicitly defines the circumscribed relation, then there is an explicit definition for the circumscribed relation which give us the desired equivalence.

Doyle also argues that, in general, minimality statements require infinitely many axioms like the first-order circumscription schema Doyle (1985). But, as proved by Theorem 3.7 and Corollary 3.8, whenever first-order logic can express a second-order Circumscription, it can be made by a single sentence. Indeed, this result is in the core of the definability results showed in this paper.

\section{REFERENCES}

BETH, E.W. "On Padoa's method in the theory of definitions". Indagtiones Mathematicae, 15, 1953.

CADOLI, M., EITER, T. and GOTTLOB, G. "Complexity of propositional nested circumscription and nested abnormality theories". ACM Transactions on Computational Logic, 6(2), p.232-272, 2005.

DAWAR, A. and GUREVICH, Y. "Fixed-point logics". Bulletin of Symbolic Logic, 8(1), p. 65-88, 2002. 
DOYLE, J. "Circumscription and Implicit Definability". Journal of Au-tomated Reasoning, 1(4), p. 391-405, 1985.

EBBINGHAUS, H.-D. and FLUM, J. Finite Model Theory. SpringerVerlag, 1995.

EBBINGHAUS, H.-D., FLUM, J. and THOMAS, W. Mathe- matical Logic. Springer-Verlag, New York, NY, 1994.

FLUM, J. "On the (infinite) model theory of fixed-point logics". In Caicedo and Montenegro, editors, Models, algebras and proofs, number 2003 in Lecture Notes in Pure and Applied Mathematics, p. 67-75. Marcel Dekker, 1999.

FERREIRA, F.M. and MARTINS, A.T. "On minimal models". Logic Journal of the IGPL, 15(5-6), p. 503-526, 2007.

HRBACEK, K. and JECH, T. Introduction to Set Theory. Marcel Dekker, 1999.

HUME, D. An Enquiry Concerning Human Understanding. 1748. Oxford University Press, 2007.

LIFSCHITZ, V. "Circumscription". In D. M. Gabbay, C. J. Hogger, and J. A. Robinson, editors, Handbook of Logic in Artificial Intelli- gence and Logic Programming-Nonmonotonic Reasoning and Uncer-tain Reasoning, V. 3, p. 297-352. Clarendon Press, Oxford, 1994.

—_. "Nested abnormality theories". Intelli- Gence, 74(2): p. 351365, 1995.

MCCARTHY, J. "Circumscription - a form of non-monotonic reasonIng”. Artificial Intelligence, 13(1-2), p. 27-39, 1980.

_. "Applications of circumscription to formalizing common-sense knowledge". Artificial Intelligence, 28(1), 1986. 
MCDERMOTT, D. and DOYLE, J. "Non-monotonic logic I". Artificial Intelligence, 13(1-2), p. 41-72, 1980.

MOINARD, Y. and ROLLAND, R. "Circumscription and definability". In International Joint Conference on Artificial Intelligence, p. 432-437, 1991.

PADOA, A. "Essai d'une théorie algébrique des nombres en tiers, precedé d'une introduction logique a une théorie deductive quelconque". In Bibliothèque Du Congrès International de Philosophie, volume 3, p. 118-123, 1900.

REITER, R. "On closed world data bases". In H. Gallaire and J. Minker, editors, Logic and Data Bases, p. 55-76, New York, 1978 .

_. "A logic for default reasoning". Artificial Intelligence, 13(1-2), p.81-132, 1980.

SCHLIPF, J. "Decidability and definability with circumscription". Annals of Pure and Applied Logic, 35(2): p.173-191, 1987.

van BENTHEM, J. "Minimal predicates, fixed-points, and definability". Journal of Symbolic Logic, 70(3): p. 696-712, 2005. 